\title{
A Conceptual Model Based on Nanoscience and Nanotechnology in Design of Smart Home for Energy Efficiency
}

\author{
P. B. Sob ${ }^{1}$, A. A. Alugongo ${ }^{2}$ and T.B. Tengen ${ }^{3}$ \\ ${ }^{1,2}$ Departmentof Mechanical Engineering, Faculty of Engineering and Technology, \\ Vaal University of Technology, Vanderbijlpark 1900, Private Bag X021, South Africa.
}

\author{
${ }^{3}$ Departmentof Industrial Engineering and Operations Management, Faculty of \\ Engineering and Technology, Vaal University of Technology, Vanderbijlpark 1900, \\ Private Bag X021, South Africa.
}

\begin{abstract}
Energy crisis and global warming are attracting the interest of the entire global community. It is therefore imperative to come up with new generation of smart homes and smart building to solve the looming energy crisis. Materials used in our homes can be designed to be more energy efficient by utilizing the principle of nanoscience and nanotechnology. This can be achieved by utilizing the classical second law of thermodynamics, the Stefan Boltzmann law of thermal radiation and also the principle of nanoscience and nanotechnology.
\end{abstract}

At nanometer dimensions the characteristic length is of the order of mean free path of electrons or phonons, and some of the laws of Newtonian mechanics in continuum can be challenged. It is therefore possible to change materials properties and behaviors at nanoscale so that the designed materials become more energy efficient when compared to their conventional counterparts that are currently used in our homes. Radiation is one of the three methods of heat transfer along with conduction and convections. The radiated heat transfer on a nanostructured material energizes the mobility of atoms in the entire systems and generates more energy in the designed nanostructured materials.

In this study a theoretical model for the application of nanoscience and nanotechnology in designing smart homes for energy efficiency has been proposed. The proposed model revealed that smart homes/buildings designed with nanomaterials are more energy efficient when compared with conventional homes designed without nanomaterials. This is a great advancement in the application of nanotechnology in smart homes for energy efficiency.

Keywords: Energy Efficiency, nanotechnology, nanoscience, smart home and building

\section{INTRODUCTION}

We do not need a rocket scientist to tell us about the current climate change and looming energy crisis. The main causes of the looming energy crisis are unclean energies (in the form of toxic gases) that are being released from industries, fuels and refrigerators from our homes [1]. Presently, researchers are more focused on clean energy for preventing destruction of ozone layer, which is for shielding out ultra-violent (UV) light from the sun. The further destruction of the ozone layer due to increase in $\mathrm{CO}_{2}$ will increase global warming [1-3]. Therefore industries must change from unclean energy to clean energy. One of such new research on clean energy is on solar power cars in automotive industries. However, most solar power designs are operating at low energy efficiency, which makes their practical applications to be limited [1-12]. Technological and engineering problems on solar system have always been focused on increasing the energy and efficiency generated from the solar systems [1-12] with limited success.

Nano-smart-home is on the spotlight since nanoscience and nanotechnology can create an industrial revolution for more efficient and clean energy [2]. Nanoscience, nanotechnology and their associated research disciplines constitute the complete spectrum of activities towards the promise of an industrial revolution [2]. The emerging disciplines of nanoscience and nanotechnology are creating the necessary experimental and computational tools for the design and fabrication of nano-dimension, electronic, photonic and energy transfer components, such as quantum dots, atomic wires, operating on nanoscopic length scales [2].

Nanoscience and nanotechnology have major impacts on several key scientific, engineering and technological activities [1-6 \& 8]. Future developments and implementation of nanotechnology could change the nature of almost every human-made object and activity [1-6 \& 8]. Think of a selfsustained home, whereby one will not pay for electricity in any of the house device (such as air conditioning system, heater, fan, fridge, cooker and TV) [3]. A home that cleans itself and windows that are designed to control the heat going in and out of the house [3] are current dreams. Nano-scientists and nanotechnologists are engaged in developing such house that will improve on our daily lifestyle [3].

The use of nanomaterials in designing new generation of smart homes will add a new form of intelligence to the build environment [3]. Materials are called nanomaterials due to reduced grain size and enhanced properties (Mechanical properties, physical properties, electrical properties, electronic properties and chemical properties) [2-6 \& 8]. Nanoscience, nanotechnology and their associated research disciplines are growing with an increasing trend in today's scientific, engineering and technological applications [2-6 \& 8]. We 
have seen recent application of nanocrystal assembly of silicon atoms manufactured by Motorola as flash memory [2]. Recent studies have shown how nanomaterials are used in medicine to produce stronger antibiotics and in the treatment of cancer [2].

The application of nanoscience and nanotechnology in designing of nano-smart-home will have more performance, energy efficiency, environmental sensitivity and sustainability [3]. This will also play a significant role on green energy [1-3 $\&$ 9-12] as well. The study of nanoscience and nanotechnology in designing of nano-smart-home for energy efficiency is therefore relevant and significant since the entire global community is currently promoting green energy and energy efficiency [1-3 \& 9-12]. Nano-smart-home has been proposed by Basma et al [3] from the construction point of view.

The design of nano smart home by Basma et al [3] has been proposed without a theoretical model, which makes reproducibility difficult or limited. The study was based on the general observation that nanoparticles are special materials with more enhanced properties. From the proposed nanosmart-home design, this current work seeks to derive a theoretical model from the application of nanoscience and nanotechnology in the design of nano-smart-home for energy efficiency. The proposed model focuses on major factors that are essential in nano-smart-home design such as thermal radiation energy, entropy in nano system, energy in nanomaterials, $\mathrm{p}-\mathrm{n}$ junction diode in solar cell, the choice of materials for the solar panel and photovoltaic system and the characterization of nanoparticles in solar system for more enhanced energy efficiency.

\section{METHODOLOGY}

\section{Theoretical Framework for Model Derivation of Nano- Smart-Home Design}

In deriving the theoretical model for the application of nanoscience and nanotechnology in designing nano-smarthome for energy efficiency, the choices of model equations for thermal radiation energy, entropy, energy in nanostructured materials, and model of $\mathrm{p}-\mathrm{n}$ junction solar cells are important. The choices of materials for the solar panel, photovoltaic systems and the characterization of nanostructured materials to boost the generation of electricity in the solar panel are also important factors in the derived model.

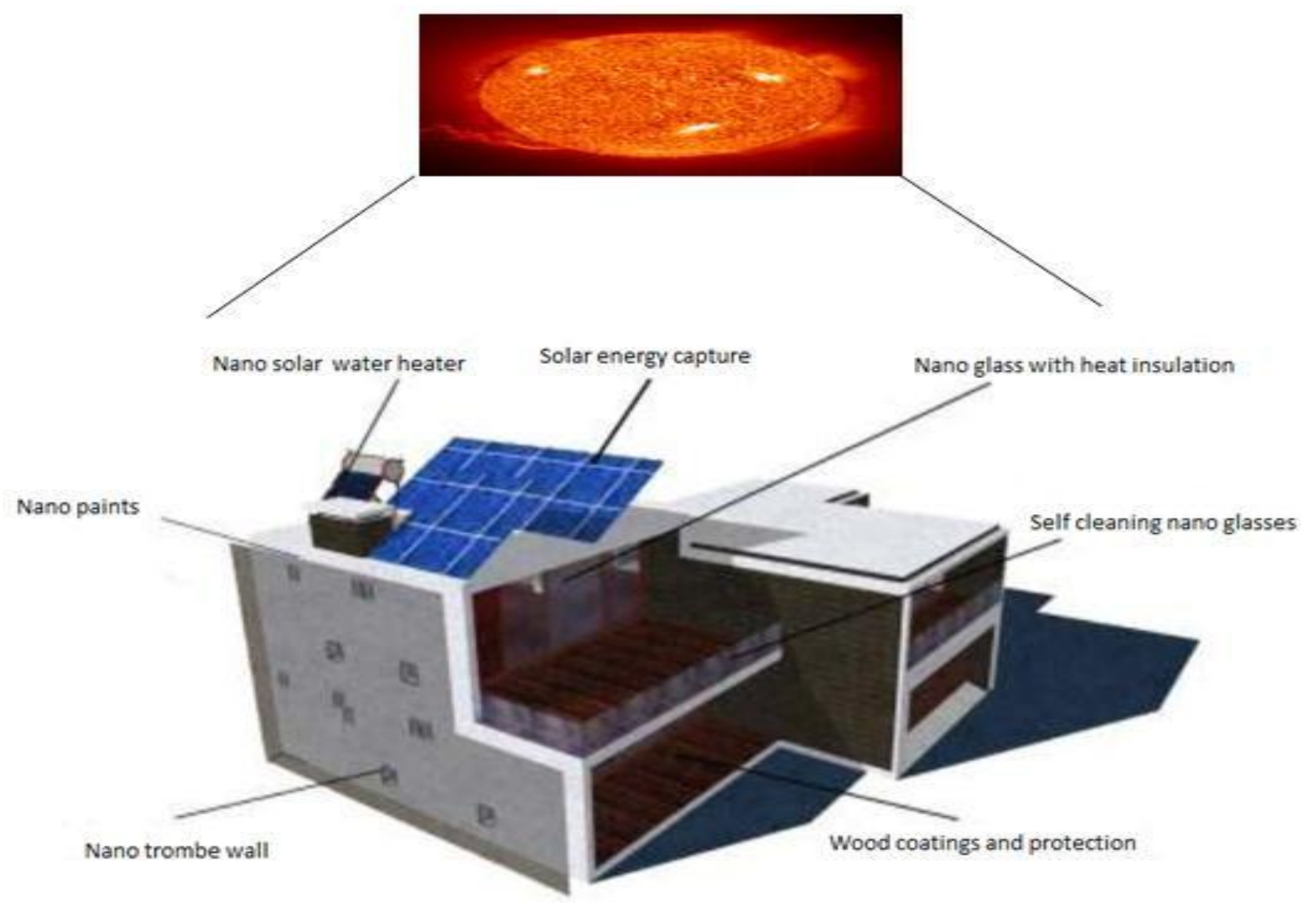

Figure 1. Schematic diagram of Nano-smart-home system (Image courtesy of [3]) 
From Fig. 1 the nano-smart home is designed to be powered by the sun. The solar panel, solar cell and other components in the house are made from nanostructured materials as indicated on the diagram. The sun light at high temperature reaches the nano-smart-home. The thermal radiation energy $P$ is given by the Stefan Boltzmann law [7] as

$$
P=e \sigma_{p} A\left(T^{4}-T_{C}^{4}\right)
$$

where $P=$ net radiated power, $A=$ radiated area, $\sigma_{p}=$ Stefan's constant, $\mathrm{e}=1, \mathrm{~T}=$ temperature of radiator, $T_{c}=$ temperature of surrounding and $\sigma_{p}=5.6703 \times 10^{-8} \mathrm{watt} / \mathrm{m}^{2} \mathrm{~K}^{4}$. Since there is change in temperature between the radiator and the surrounding it is important to understand the heat content of materials in nano-smart-home since the proposed nano-smarthome is designed from nanomaterials. The definition of entropy due to the flow of heat in a material and heat released to the surrounding of the material, which was originally developed for macro systems, is also valid for nano systems [2] but with modifications. It should be noted here that, the flow of heat in a materials and heat released to the surrounding of the material were due to the increase in temperature of radiator and the temperature of the surrounding. In nano system the term entropy, S, may assume a new statistical form due to nonextensivity of nanostructured materials [2]. The expression of the change of entropy is given by [2] as

$d P_{s}=d S-\frac{d Q_{i n}}{T_{e x t}}$

where $d S$ the change of entropy, $\quad d P_{s} \quad$ is the differential entropy in a closed system, $d Q_{i n}$ is the heat supplied $T_{e x t}$ is the external temperature. Since there is flow of heat in nanostructured materials due to thermal radiation energy, there is a possibility of change of energy in the material. The stochastic model of energy $d E$ in nano crystalline materials as derived by [8] is given as

$$
\begin{aligned}
& \mathrm{dE}=\frac{\delta^{\underline{4}} \pi \mathrm{r}^{3}}{\mathrm{~N} \delta_{3}^{\underline{4}} \pi \mathrm{r}^{3}}+\frac{\mathrm{N} \delta\left(\begin{array}{l}
\underline{4} \pi \mathrm{r}^{3}-\underline{4} \\
3 \mathrm{r}^{3}
\end{array}\right)}{\mathrm{N} \delta^{\frac{4}{\pi}} \mathrm{r}^{3}} \\
& -\left(T_{\text {ext }} d S-d P_{S} T_{\text {ext }}\right)+\sigma r^{2} d r
\end{aligned}
$$

where $r_{0}$ is the initial grain size, $\delta$ is the surface tension, $r$ is the equivalent radius of the grain during deformation of nanostructure materials, $N$ is a constant from the overall number of atoms in the given sphere, $\sigma$ is the yield stress and $d r$ is the change of size during deformation of nanostructure materials. Equation (1) gives the radiated power emitted into nano solar panel and nano-smart-home. Equation (2) gives the differential entropy in a closed nano system when the net radiated power is emitted into the nano solar panel and nano-smart-home. Equation (3) gives the stochastic model of energy in nanomaterials. The next step is to study the choice of materials for the solar panel and photovoltaic system and also the total power being generated by the $p-n$

junction solar cells and the theory and future trends of nano smart home design.

\section{The Choice of Materials for the Solar Panel and Photovoltaic System}

Aluminum plays a vital role as one of the key materials widely used in renewable energy systems (solar thermal collectors, wind turbines, photovoltaic systems, solar cookers and concentrating solar thermal power plants) [9-12]. Studies support the increased trend of aluminum as absorbers in solar system [9-12]. Recent research finds have also shown that $0.4 \mathrm{Mt}$ of aluminum is used in PV system today [9]. However, the main reasons for the use of aluminum in solar cell metallization are due to its low cost and good electrical properties [9-12]. Besides the fact that aluminum is a good electrical conductor, aluminum also forms a low-resistivity contact to $\mathrm{p}$-and n-doped silicon [10]. Another great advantage of aluminum is that it has a very good reflection property, which is key and very important for IBC solar cells. The above advantages make aluminum a vital material for new generation solar panel systems.

Temperature is an important factor when studying the efficiency of photovoltaic solar systems [9-12]. Research findings have revealed that increasing temperature reduces the efficiency of PV solar cells [9-12]. It is therefore imperative to set a cooling system for the PV. This makes the use of aluminum in PV cell very unique due to the fact that it is very easy to reduce the temperature of aluminum material [12]. The use of aluminum in solar power for electricity generation will greatly be more efficient base on the designing, doping and characterization process [9-12].

\section{P-N Junction, the Theory and Future Trends in Solar Cells}

Electromagnetic radiation is normally emitted from the sun, which is then absorbed by the solar panel, nano-smart-home and nano solar cell. A photon will then excite a negatively charged electron from the valence band (low energy state) to the conduction band (a higher energy state) leaving behind a positively charged vacancy, called a hole [4]. For this energy transfer to create any usable energy, the photon must have energy greater than the band gap of the material, or else the excited electron will immediately relax down and recombine with the hole and the energy will be lost as heat [4].

Upon the excitation in the band gap, the photon creates an electron and a hole which are now free to move throughout 
the semiconductor crystal. These acts as charge carriers that transport the energy to the electrical contacts, which results in a measurable external current. The described processes are shown in Fig. 2 (a-d).

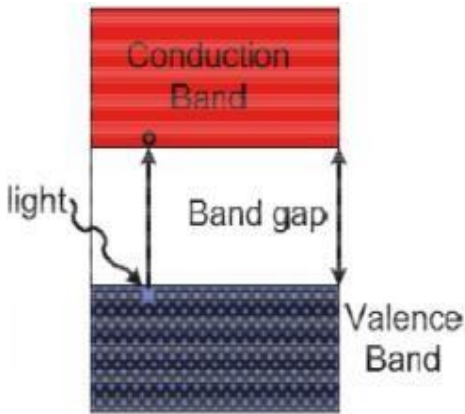

(a)

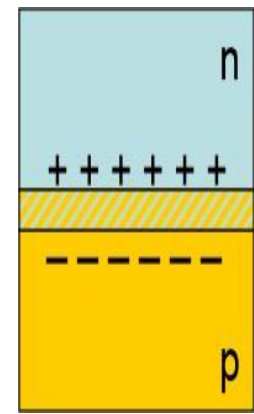

(b)

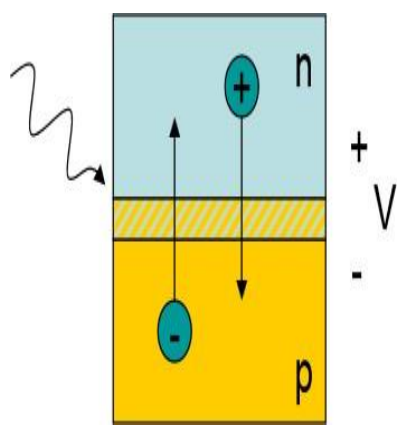

(c)

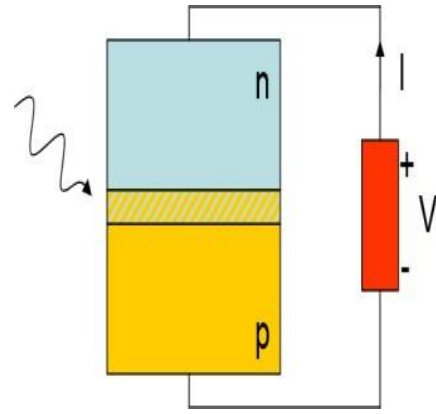

(d)

Fig. 2. Light Conversion in Process in Solar Cell (Image courtesy of [4])

The solar cell made from nanomaterials' contains more atoms, electrons and holes that move at a higher rate. When materials are at nanoscales the mobility of their atoms increases at a higher rate when compared with conventional materials [1]. Generally materials at nanoscales have more enhanced properties (mechanical or electrical properties) [1, $6 \& 8$ ]. Since the mobility atoms at nanoscales are higher, their vibration energy becomes greater as they mover at a higher rate creating more holes which acts as charge carriers. Since there are more electrons in nanomaterials, more holes are generated in nanomaterials' therefore more electrons jump across the band gap and more holes are created at the lower energy band as more charges are said to be generated in a nanomaterials' when compared with conventional materials [1, $6 \& 8]$. The current generated is more since there are more electrons create more holes at nanoscales when compared with conventional materials. These are the reason that makes nanomaterials' more energy efficient as an electrical conductor when compared with conventional materials. Hence were ever nanomaterials' are used as an electrical conductor the electrical conductivity of the materials generally increases. Since our theoretical derived model must be simulated with the band gap energy, it is important to consider the band gap energy given by [4] as.

$$
E=h\left(\begin{array}{l}
C \\
\lambda
\end{array}\right)
$$

where $h=$ Planks constant $=6.626 \times 10^{-34}$ Joules sec, $C=$ speed of light $=3.0 \times 10^{8}$ meter $/ \mathrm{sec}, \lambda=$ cut off wavelength $=410.57 \times 10^{-9}$ meters and $E=$ band gap energy.

The total solar cell efficiency is given by [4] as

$\eta=\frac{P_{\max }}{E_{S, \gamma}^{S W} A}$

where $E_{S, \gamma}^{S, W}$ is solar irradiance in $\mathrm{W} / \mathrm{m}^{2}$ under standard conditions $=1000 \mathrm{~W} / \mathrm{m}^{2}, P_{\max }=400 \mathrm{~W}$. The radiated area is derivable from the area or volume of 3-D grain in nanostructured materials, which can be related to the equivalent volume of 3-D grains through $\frac{4}{3} \pi r^{3}=V={ }_{\frac{4}{3}}^{4} \underset{123}{r} \underset{3}{r}$. This leads to consideration of the different models of size variants for 3-D grains that have been previously defined by [6] and are used in this report, which are,

$$
d r_{1}=M\left(\frac{1}{-\frac{1}{r_{c 1}}-r_{1}}\right) d t+r_{1}{ }^{2} D d W(t)-Z r_{1} V_{1} d(t)
$$

where $\langle\ldots\rangle=$. the expected value, $r_{c l}=$ local critical grain size, $Z$ and $D$ are Constants, $d W(t)=$ change of the Wiener process, $V_{1}=\tau_{1} r^{2}$ defines rate of grain breakage or number of coalescence, events within an infinitesimal time interval,

$$
M=M_{0}\left(1+\frac{C D}{r_{1}}\right) \text { is Grain Boundary (GB) mobility }
$$

function, $\quad C D=4(H m)\left(h_{0}\right) /((k)(T)), \quad T_{m}=T\left\{\ln \left(m_{01} / m\right) \quad\right.$ and $M_{0}=M_{01} \exp \left\{-T_{m}(\right.$ inf $\left.) / T\right\}=$ initial grain boundary mobility constant.

$$
\begin{aligned}
& d r_{3}=\operatorname{Ratio}_{1}\left(d r_{1}\right) \\
& d r=-O r d t+I d W(t)
\end{aligned}
$$

where $O$ and $I$ are constants.

$$
d r=\text { Ratio }(d r)
$$

The stochastic counterparts of equation (1) to (9) are considered so as to take care of their random fluctuations inherited from the random grain sizes in the nanomaterials used in the nano-smart-home. The resulting equations are solved simultaneously using Engineering Equation Solver software (F-Chart Software, Madison, W153744, USA). 


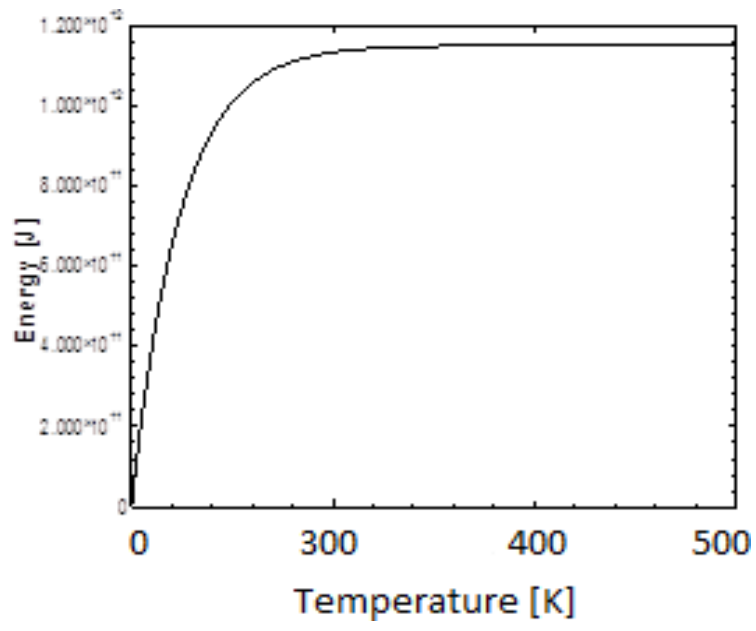

(a)

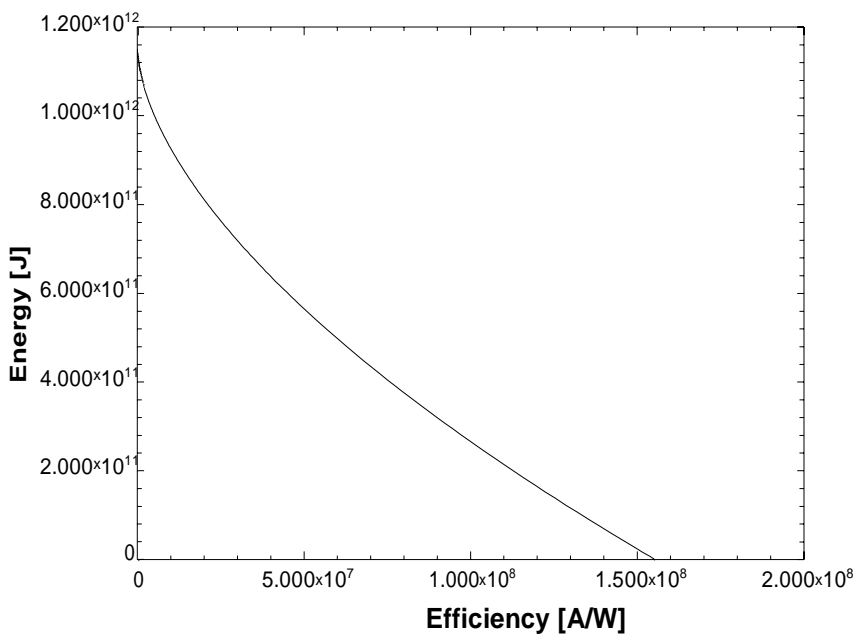

(b)

Figure 3. (a) Energy [J] against Temperature [K] (b) Energy [J] against Efficiency [A/W]

\section{RESULTS AND DISCUSSION}

The proposed models derived in this paper are tested with the data from (nanocrystalline) aluminum sample [14] which are $M_{0}{ }^{\prime}=0.01 \mathrm{~nm}^{2} \mathrm{~s}^{1}, \quad m=4, \quad r_{c l}=1.95 \mathrm{r}, \quad C C=12, \quad a=0.90, \quad D=10^{4}$, $h_{0}=0.25 \mathrm{~nm}, T_{m}(\infty)=933.47 \mathrm{~K}, C V_{0}=0.3, H_{m}(\infty)=10.71 \mathrm{KJMol}^{1}$, $\sigma_{0}{ }^{\prime}=16.7 \mathrm{MPa}, \quad K_{t}=1.3, \quad \sigma_{0}=15.40 \mathrm{MPa}$, $K d=1301.77 \mathrm{MPa} \_n m^{1 / 2}, \quad R=8.31 \mathrm{JK}^{-1} \mathrm{~mol}^{-1}$ and $T_{r}=300 \mathrm{~K}$, $K_{B}=1.381023 \mathrm{~J} / \mathrm{K}$. The additional data obtained for this work are $O=0.0035, I=1.1, r_{0}=100 \mathrm{~nm}, Z=0.4$, Ratio $_{I}=0.81$, Ratio $_{2}=1.071$, and $\tau_{1}=0.000008$. The additional data were obtained through curve fitting of the empirical data from the different measurements of the sizes. The obtained results are presented in the plots above.

The proposed design of a conceptual model for the application of nanoscience and nanotechnology for nanosmart home from Fig. 1 have several major parameters as indicated in Fig. 1 that will be consider in future modeling and simulations. In this current paper we focus on energy, efficiency, temperature and the radiated power on the nanosolar panel in nano-smart-home design. Since our main objective of this project is derive a theoretical model for a smart-home from nanomaterials' which will facilitate the reproducibility of a modern solar panel for a nanosmart home. The obtained results from theoretical modeling and simulation of the total energy produced from solar panel systems shown improved results and efficiency. The total energy and efficiency are said to be more improved when compared with a solar panel produced from conventional materials. The results are of great significant since it has shown significant energy and efficiency. It has been revealed as shown in Fig. 3 (a) that, the energy increases with increase in temperature in nano-smarthome design. It has also been revealed from modeling as shown in Fig.3 (b) that the efficiency will drop at high energy level in nano-smart-home. The drop in efficiency is due to the fact that at high energy level the temperature of the system (PV cell) has significantly increased which negatively affect the efficiency of nano-smart-home. Temperature is an important factor when modeling and designing of nano-smarthome for energy efficiency.

Modeled results have revealed that, as the energy increased with increase in temperature, there existed a high energy level that the efficiency in the system started dropping since the temperature had exceeded the acceptable temperature in nano PV solar cells. This emphasizes importance of designing nanosolar panel system from nanocrystalline aluminum due to the fact that it is very easy to reduce the temperature of aluminum material.

Nanocrystalline materials are generally unstable at very high temperature, as several studies have revealed that continuous increase in temperature has a negative impact on nanocrystalline materials properties [6 \&13]. The increase in energy can be explained due to the fact that at nanoscales there is an increase in mobility of atoms, holes and free electrons that lead to an increase in energy as shown in Fig. 3 (a). The figures below revealed the variations of efficiency against temperature and radiated power on the design nanosmart-home. 


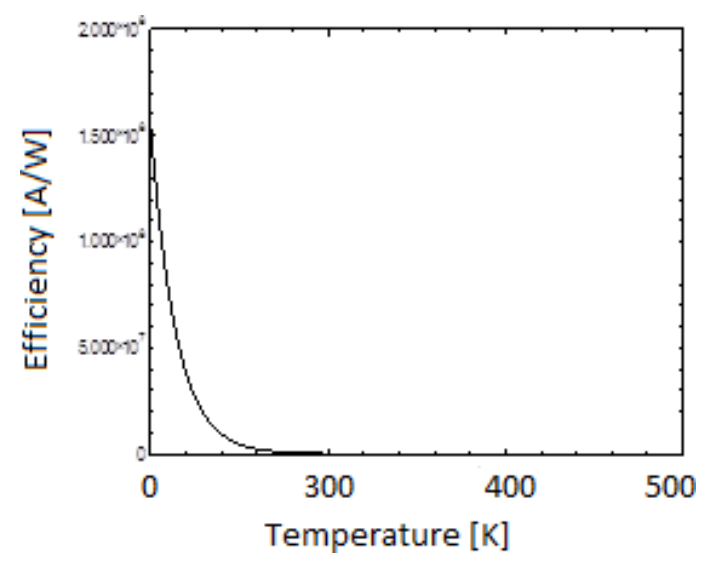

(a)

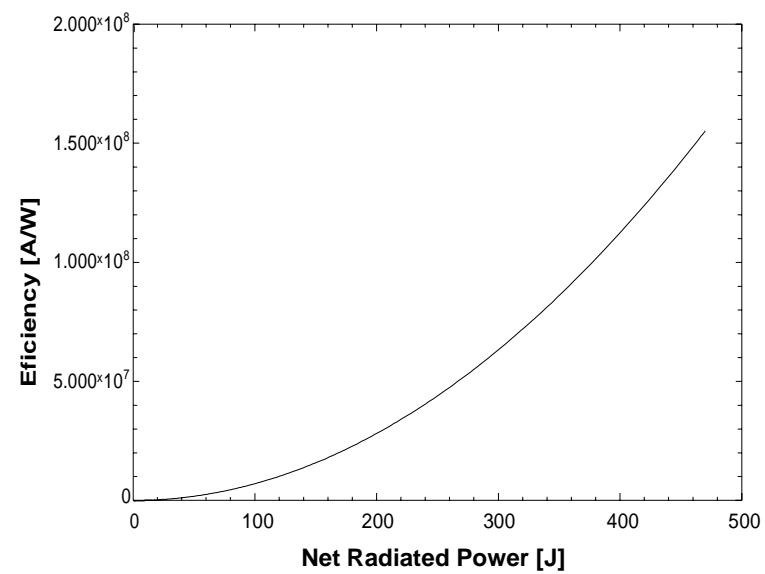

(b)

Figure 4. (a) Efficiency [A/W] against Temperature [K] (b) Efficiency [A/W] against Net Radiated Power [J]

Modeled results revealed that the efficiency in the system decreases with increase in temperature as shown in Fig. 4 (a) while the efficiency in the system increases with increase in net radiated power as shown in Fig. 4 (b). It is observed from the above results that, energy and efficiency in nano-smarthome drop at extremely high temperature. Therefore, to maintain a steady energy and efficiency in the designed nanosmart-home, provision for cooling the solar panel system is very important for the system to be more energy efficient. The theoretical model derived for the application of nanoscience and nanotechnology for nano-smart-home revealed that the system will be more energy efficient if designed with thermal cooling system for the PV.

The success and application of nanoscience and nanotechnology in designing smart homes for energyefficiency greatly depend on the ability to synthesize and to characterize nano particles for proper understanding of their properties (physical, mechanical, electrical and chemical). The study of synthesis of nano particles for nano-smart-homes and the integration of the study for the design of nano smart home with more energy and efficiency is in progress by the same authors. This is a great progress and advancement in the application of nanotechnology in smart homes for energy efficiency.

\section{CONCLUSION AND RECOMENDATION}

The study was aimed at proposing a theoretical model for the application of nanoscience and nanotechnology in designing of smart homes for energy efficiency. This was achieved by utilizing the classical second law of thermodynamics, the Stefan Boltzmann law of thermal radiation and also the principle of nanoscience and nanotechnology.

It was observed that the energy increases with increase in temperature. It was also observed that there exists a high energy level that the efficiency in the system started dropping, since the temperature exceeded the acceptable temperature in nano PV solar cells. It was also observed that, the efficiency in the system increases with increase in net radiated power. The models proposed in this study focused on parameters or variables that can be measured theoretically and can be validated experimentally. Therefore it will be proper to focus on the prototype project which will be the next phase of the current research.

\section{ACKNOWLEDGEMENT}

This material is based on the work which is supported financially by the National Research Foundation (NRF) and Vaal University of Technology (VUT).

\section{REFERENCE}

[1] Xiaohu H. Guozhong, X. Yongfeng L. \& Ekaterina N. Nanomaterials' for Energy-Efficient Applications Journal of Nanomaterials. Volume 2015, Article ID 524095 .

[2] Ali Mansoori. G., "Principles of nanotechnology: Molecular-Based Study of Condensed Matter in Small Systems", World Scientific Publishing Co. Pte. Ltd.5 TohTuch Link, Singapore 596224, 2004.

[3] Basma, H. Ghada, H. Menna, A. \& Mona, B. M. Nano Smart Home an Interdisciplinary Collaboration for a Better Quality of the Build Environment. 2 $2^{\text {nd }}$ International Conference on Energy Systems and Technologies 18 - 21 Feb. 2013, Cairo, Egypt

[4] Suresh, S. Recent trends on nanostructures based solar energy applications: A review. Rev. Adv. Mater. Sci. 34 (2013) 44-61

[5] Richa, S. Investigation on Temperature Sensing of Nanostructured Zinc Oxide Synthesized via Oxalate Route. Journal of Sensor Technology, 2012, 2, 8-12 
[6] Sob, P. B. Alugongo, A. A. Tengen, T. B. "Modelling Strain Rate Sensitive Nanomaterials' Mechanical Properties: the effect of varying definitions, M. Tech. Dissertation. Vaal University of Technology, 2016

[7] Mark, W. The Stefan-Boltzmann Law Physics Department, The College of Wooster, Wooster, Ohio 44691, USA : May 9, 2007

[8] Sob, P. B. Alugongo, A. A. Tengen, T. B. Stochastic Model of Nanocrystalline Materials Energy Produced by Accumulative Roll-Bonding. 10 ${ }^{\text {th }}$ South African Conference on Computational and Applied Mechanics Potchefstroom 3-5 October 2016

[9] Martin, P. Aluminium and Renewable Energy Systems Prospects for the Sustainable Generation of Electricity and Heat. Institut für Energie und Umweltforschung Heidelberg GmbH 2010

[10] Schroder, D. K. \& Meier, D. L. "Solar cell contact resistance - a review", IEEE Transactions on Electron Devices, ED-31, 5, pp. 637-647, 1984.

[11] Mathias, K. \& Jonas, B. \& Roman, K. \& Mike, J. \& Ralph, M. \& Markus, G, \& Stefan, W. \& Glunz, \& Ingo, K. Plating processes on aluminum and application to novel solar cell Concepts. 4th International Conference on Silicon Photovoltaics, Silicon PV 2014

[12] Amir, F. \& Maysam, M. \& Zaki, A. \& Intesar, A. Aluminium Alloys in Solar Power - Benefits and Limitations

[13] Sob, P. B. Alugongo, A. A. Tengen, T. B. The Effects of the Size Variants of Nanocrystalline Materials Produced by Accumulative Roll-Bonding on their Energy, Thermodynamics and Mechanical Properties. $10^{\text {th }}$ South African Conference on Computational and Applied Mechanics Potchefstroom 3-5 October 2016

[14] TENGEN, T. B. 2008. Analysis of Characteristic of Random Microstructures of Nanomaterials PhD. Thesis. Witwatersrand Johannesburg. 